\title{
Long-Term Changes in Corneal Structure and Tear Inflammatory Mediators after Orthokeratology and LASIK
}

\author{
Javier González-Pérez, ${ }^{1,2}$ César Villa-Collar, ${ }^{1,3}$ José Manuel González-Méijome, ${ }^{1,4}$ \\ Nery García Porta, ${ }^{1}$ and Manuel Ángel Parafita ${ }^{1,5}$
}

Purpose. To monitor changes in corneal structure and level of inflammatory mediators in tears of myopic patients following orthokeratology (OK) and laser in situ keratomileusis (LASIK).

Methods. Sixty-two myopic subjects were analyzed in this prospective study. Thirty-two had worn Paragon CRT contact lenses and 30 underwent standard LASIK. Thirty-two control subjects were also recruited. Pachometry and corneal topography were performed 12 months after both interventions. Tears were collected and assayed using ELISA for IL-6, IL-8, matrix metalloproteinase-9 (MMP-9), and epidermal growth factor (EGF).

RESults. Corneal power decreased in the central area and increased in the paracentral area. Simultaneously, there was a central corneal thinning with mid-peripheral thickening for OK. No changes were observed for LASIK. In comparison with controls, IL-6, IL-8, MMP-9, and EGF levels were increased significantly for OK $(P<0.01)$. Only MMP-9 $(P<0.01)$ and EGF $(P<0.01)$ were increased for LASIK. Pro-inflammatory response was found to be associated with the degree of myopia in the OK group. The MMP-9 and EGF upregulation was linked to the presence of iron deposition in both groups, whereas increased levels of EGF were connected with the presence of myopic regression after LASIK.

Conclusions. Both interventions involved important tissue reshaping. The physical presence of a reverse geometry contact lens caused certain markers to change significantly in comparison with no lens wear or LASIK in the long-term response. MMP-9 was significantly higher in tears of subjects presenting iron deposition after OK or LASIK, and EGF levels seemed to play an important role in postoperative refractive outcomes after LASIK. (Invest Ophthalmol Vis Sci. 2012; 53:5301-5311) DOI:10.1167/iovs.11-9155

From the ${ }^{1}$ Ocular Surface and Contact Lens Research Group, ${ }^{2}$ Department of Applied Physics (Optometry), and ${ }^{5}$ Department of Surgery (Ophthalmology), Universidade de Santiago de Compostela, A Coruña, Spain; ${ }^{3}$ Clínica Oftalmológica Novovisión, Madrid, Spain; and ${ }^{4}$ Clinical and Experimental Optometry Research Laboratory, Center of Physics (Optometry), School of Sciences, University of Minho, Braga, Portugal.

Supported by National Grant PI081380 from Instituto de Salud Carlos III, Spain, awarded to the Ocular Surface and Contact Lens Research Group GI-1750.

Submitted for publication November 24, 2011; revised April 19 and June 9, 2012; accepted July 2, 2012.

Disclosure: J. González-Pérez, None; C. Villa-Collar, None; J.M. González-Méijome, None; N. García Porta, None; M.Á. Parafita, None

Corresponding author: Javier González-Pérez, Ocular Surface and Contact Lens Research Group, Department of Applied Physics (Optometry), Universidade de Santiago de Compostela, 15782 Santiago de Compostela, A Coruña, Spain; javier.gonzalez@usc.es.
There are different surgical and nonsurgical strategies to 1 correct myopia, including orthokeratology (OK) and laser in situ keratomileusis (LASIK).

Modern OK involves the use of high-Dk rigid gas-permeable (RGP) lenses worn on an overnight basis. The main benefit consists of dispensing with any kind of optical correction during the day that might interfere with professional, sports, and recreational activities. ${ }^{1}$ Recent studies have shown the method to be effective in slowing down the progress of myopia, which contributes to an increased use of $\mathrm{OK}^{2}$ This treatment induces flattening of the corneal curvature and redistribution of corneal tissue, ${ }^{3}$ the central corneal epithelium grows thinner, while the mid-peripheral corneal epithelium thickens. To the best of our knowledge, there are no previous studies on how pro-inflammatory molecules, matrix proteases, and growth factors could be affected during this tissue redistribution.

LASIK is a surgical procedure that involves creating a thin corneal flap with a microkeratome followed by excimer laser ablation of the stroma. This surgical procedure is considered relatively safe and is followed by low or mild inflammation in the 24 hours after surgery. ${ }^{4}$ The cellular, molecular, and neural regulatory phenomena associated with postoperative inflammation and wound healing are likely to be involved in the rare adverse effects of LASIK, such as flap melt, epithelial ingrowth, and myopia regression.

The clinical efficacy of both techniques for reduction of low to moderate myopia is now becoming well established, but new studies are necessary to shed light on the underlying corneal events associated with the significant central corneal flattening induced by reverse geometry lenses and LASIK. Although nonsurgical and surgical procedures aim to modify the refractive status of the eye by intervening on the anterior surface of the cornea, the ways to achieve this goal are quite different. OK aims at a redistribution of the corneal tissue through the effect of overnight contact lens wear (persistent stimulation), whereas LASIK aims at the removal of the tissue within the ablation area (acute stimulation), leaving the peripheral cornea apparently unchanged. This can also lead to a significantly different final result with regard to the shortand long-term corneal inflammatory response, consequently, with regard to cytokine-, chemokine-, matrix protease-, and growth factor-mediated effects. It is important to analyze whether these effects may constitute determinant factors for the development of different corneal complications that may occur with LASIK or OK in the treatment of myopia, hyperopia, or astigmatism. ${ }^{1,4}$

Epithelial cells and keratocytes have receptors for different cytokines, chemokines, and growth factors. Thus, the activity of inflammatory mediators in these cells may be important for the response of corneal tissue to wounding and tissue redistribution. ${ }^{5}$ Several studies have focused on these tear proteins, which are known to regulate wound healing, apoptosis, cell cycling, and migration on the ocular surface 
in physiological, post-contact lens wear, post-surgical, and pathological conditions. ${ }^{6-14}$

The aim of the present study was to evaluate a pattern of molecules involved in corneal response and the wound-healing process in tear samples obtained from myopic subjects after 12 months of overnight OK and LASIK. This was intended to provide insights into the comparative long-term changes experienced by both myopia treatments involving several mediators in the corneal wound-healing response in a similar cohort.

\section{Materials ANd Methods}

\section{Patient Selection}

Sixty-two healthy myopic subjects ( 30 females and 32 males), aged 30.0 \pm 6.5 years, participated in this cross-sectional study. Thirty-two subjects ( 16 females and 16 males) aged $27.0 \pm 7.4$ years had worn OK contact lenses on an overnight basis for 12 months. This study also comprised 30 myopic patients (14 females and 16 males), aged $33.1 \pm$ 5.7 years, who had undergone LASIK at the Ophthalmology Clinic Novovision (Madrid, Spain). Thirty-two subjects (16 females and 16 males) aged $26.7 \pm 5.1$ years who have never worn contact lenses were also recruited as the control group.

Only patients with myopia between $-1.00 \mathrm{D}$ and $-6.00 \mathrm{D}$ and astigmatism below $-1.75 \mathrm{D}$ were included, in agreement with the Food and Drug Administration-approved range of refractive errors treated with OK. All subjects were asymptomatic, and before contact lens fitting were examined to discard the presence of any inflammatory disease. Patients completed the modified McMonnies dry-eye symptom questionnaire; the tear break-up time (TBUT) was evaluated 1 minute after instilling $5 \mu \mathrm{L}$ of $1 \%$ sodium fluorescein (Chauvin Pharmaceutical, Aubenas, France). Last, the tear production test was performed using Schirmer test strips (Chauvin Pharmaceutical). Inclusion criteria for tear sample collection included a symptom severity score lower than 14, TBUT more than 7 seconds, and Schirmer test greater than $15 \mathrm{~mm}$ A minimum of 3 months after treatment was required to guarantee that the topography was completely stable. ${ }^{15,16}$ Tear samples, corneal thickness, and curvature were examined 12.03 (range 6-16) and 10.80 (range 6-13) months after OK and LASIK treatments respectively. The exclusion criteria were the presence or history of any systemic or ocular disorder, including ocular surgery, trauma, or disease.

\section{Corneal Refractive Therapy Lens Characteristics}

Reverse geometry RGP lenses were used for the OK treatment (Paragon Vision Sciences, Mesa, AZ). Trial lenses were derived from nomograms in the form of sliding tables produced by the manufacturer of the Paragon CRT sigmoid reverse geometry contact lenses. ${ }^{17}$

\section{LASIK Procedure}

The same surgeon performed all LASIK procedures using the same standard surgical technique. After creating a $120-\mu \mathrm{m}, 9.5-\mathrm{mm}$ diameter flap with a Hansatome microkeratome (Chiron Vision, model 2765; Bausch \& Lomb, Claremont, CA), standard ablation profile was produced using the Allegretto Wave Eye-Q $400 \mathrm{~Hz}$ (Wavelight Laser Technologie, Erlangen, Germany). The type of ablation was central, with an optic zone of $6.50 \mathrm{~mm}$ for all cases. All surgical procedures were uneventful and considered successful.

\section{Ethical Considerations}

The Ethics Committee of the Santiago de Compostela University approved this study, and all procedures involving human subjects were in compliance with the Declaration of Helsinki. Informed consent for tear collection, contact lens fitting, or refractive surgery was obtained from all subjects after explaining the possible consequences of each procedure.

\section{Corneal Thickness and Curvature}

Central and peripheral corneal thickness was obtained with Scheimpflug Pentacam (Oculus, Wetzlar, Germany), previously calibrated using the test samples provided by the manufacturer. Measurements were recorded at three different radial distances of 1 , 2 , and $3 \mathrm{~mm}$ from the corneal center along eight semi-meridians located at $45^{\circ}$ intervals $\left(0^{\circ}, 45^{\circ}, 90^{\circ}, 135^{\circ}, 180^{\circ}, 225^{\circ}, 270^{\circ}\right.$, and $\left.315^{\circ}\right)$. To minimize the effect of diurnal corneal hydration changes on corneal thickness, all measurements were performed in the afternoon between 2:00 and 6:00 PM. ${ }^{18}$

\section{Tear Sample Collection}

Tear samples were collected as described previously, using $50 \mu \mathrm{L}$ of calibrated disposable glass microcapillaries (Micro Titertube natural 845-TP; TTE Laboratories, Hopkinton, MA) from the inferior temporal tear meniscus at the lower lid margin. From each subject, 5 to $15 \mu \mathrm{L}$ of tears was collected in 5 minutes. Care was taken to avoid touching the lid margins or the corneal surface. While collecting tears, flow rate was controlled, and only samples with a flow rate of 1 to $7 \mu \mathrm{L} / \mathrm{min}$ were used in order to avoid the dilution effect caused by a possible hypersecretion of tears during the process. Microcapillary tubes were immediately transferred to Falcon tubes and stored at $-80^{\circ} \mathrm{C}$ for subsequent determination of inflammatory molecules.

\section{Assays for Inflammatory Mediators}

Tears were assayed for IL-6, IL-8, matrix metalloproteinase 9 (MMP-9), and epidermal growth factor (EGF). The concentrations of inflammatory mediators were measured with commercially available quantitative sandwich ELISA kits (Quantikine; R\&D Systems Europe, Abingdon, UK) following the protocols supplied by the manufacturers. The samples were centrifuged at $13,000 \mathrm{rpm}$ for 5 minutes and supernatant transferred to new Falcon tubes. Since tear samples were available in small volumes, each sample was diluted 1:20, 1:80, and 1:100 for cytokines, EGF, and MMP-9 respectively, with sample calibrator diluents (reagents provided with each ELISA kit). The final results were adjusted according to the dilution factor. Accurate sample concentration was calculated from the linear correlation made with standard antigen concentration versus absorbance. All determinations were carried out without the technician's knowledge of the sample characterization (blind test).

\section{Statistical Analysis}

Data were analyzed using the statistical package for social sciences: SPSS 18.0 (SPSS Inc., Chicago, IL). The Kolmogorov-Smirnov normality test was applied to assess all data sets for normal distribution. ANOVA and Mann-Whitney test were used to check for potential differences in baseline data between two treatment groups for normally or nonnormally distributed variables, respectively. This allowed us to establish whether the treatment groups were comparable or not in terms of baseline values. Paired sample $t$-test was used to compare corneal thickness and curvature data between pre- and post-treatment in each group. Because the tear marker's data sets were not normally distributed, Mann-Whitney tests were used to compare differences in cytokine, MMP-9, and EGF concentrations in subjects undergoing OK or LASIK treatments versus controls. Kruskal-Wallis analysis was used to check for potential differences regarding the amount of refractive error in subjects undergoing surgical and nonsurgical treatments as per comparison with control data. The minimal level of confidence at which results were deemed significant was established at $P<0.05$.

\section{RESULTS}

Both groups were matched in terms of baseline age, refractive error, and duration of treatment. No statistically significant 
TABLE 1. Descriptive Statistics (Mean \pm SD) for the Sample at the Start of the Study

\begin{tabular}{|c|c|c|c|c|c|}
\hline \multirow[b]{2}{*}{ Case-Control } & \multirow{2}{*}{$\frac{\text { Control }(n=32)}{\text { Median } \pm \text { SD }}$} & \multicolumn{2}{|c|}{ OK $(n=32)$} & \multicolumn{2}{|c|}{ LASIK $(n=30)$} \\
\hline & & Median \pm SD & $\boldsymbol{P}$ & Median \pm SD & $\boldsymbol{P}$ \\
\hline Age, $y$ & $26.72 \pm 5.12$ & $27.00 \pm 7.38$ & $0.855^{*}$ & $33.12 \pm 5.67$ & $<0.001^{*}$ \\
\hline Initial sphere, D & $-2.57 \pm 1.34$ & $-2.58 \pm 1.04$ & $0.537 t$ & $-2.77 \pm 1.01$ & $0.346 \dagger$ \\
\hline Initial cylinder, D & $-0.50 \pm 0.42$ & $-0.25 \pm 0.33$ & $0.036 t$ & $-0.58 \pm 0.50$ & $0.039 \dagger$ \\
\hline Spherical equivalent, D & $-2.76 \pm 1.28$ & $-2.69 \pm 1.07$ & $0.951 \dagger$ & $-2.99 \pm 1.03$ & $0.312 \dagger$ \\
\hline CC center, D & $43.87 \pm 1.57$ & $43.89 \pm 1.84$ & $0.957^{*}$ & $43.36 \pm 1.37$ & $0.221^{*}$ \\
\hline $\mathrm{CC} \operatorname{cord} 2 \mathrm{~mm}, \mathrm{D}$ & $43.54 \pm 1.70$ & $43.64 \pm 1.72$ & $0.827^{*}$ & $43.14 \pm 1,17$ & $0.311^{*}$ \\
\hline $\mathrm{CC}$ cord $4 \mathrm{~mm}, \mathrm{D}$ & $42.93 \pm 1.56$ & $43.04 \pm 1.77$ & $0.807^{*}$ & $42.76 \pm 1.01$ & $0.627^{*}$ \\
\hline CC cord $6 \mathrm{~mm}, \mathrm{D}$ & $41.63 \pm 1.31$ & $41.64 \pm 1.73$ & $0.533^{*}$ & $41.89 \pm 1.07$ & $0.115^{*}$ \\
\hline Time of study, mo & - & $12.03 \pm 2.06$ & - & $10.80 \pm 2.02$ & $0.075 \dagger \neq$ \\
\hline$n$ (sample) & 32 & 32 & 1.000 & 30 & $0.082 \dagger$ \\
\hline
\end{tabular}

CC, corneal curvature.

* ANOVA (Bonferroni post hoc)

† Mann-Whitney test.

‡ Data obtained among LASIK and OK groups.

differences were found between groups for the initial refractive error in terms of sphere and mean spherical equivalent, keratometric radius, or corneal thickness $(P>$ 0.05). Table 1 shows the subject demographics and the refractive status of right eyes at baseline. No adverse events were observed with the exception of some minor changes, such as trace to moderate corneal staining in the OK group, the presence of pigmented deposition in both OK and LASIK patients, and some cases of myopic regression in the LASIK group. These findings will be used to correlate their presence with the upregulation of certain molecules under analysis.

\section{Corneal Thickness and Curvature}

The average baseline central corneal thickness and curvature for the OK group was $560 \pm 29 \mu \mathrm{m}$ and $43.89 \mathrm{D}$, compared with $551 \pm 33 \mu \mathrm{m}$ and $43.36 \mathrm{D}$ in LASIK patients. As expected, paired $t$-test showed statistically significant differences between baseline values and those obtained after 12 months of OK and LASIK in corneal curvature and thickness for the central and mid-peripheral regions. Tables 2 and 3 shows mean value, $\mathrm{SD}$, and the value of statistical significance for corneal curvature and thickness differences between pre- and posttreatment for the OK and LASIK groups. These tables show a statistically significant flattening of the central cornea $(P<$ 0.001 ) measured as local corneal power decrease in the $2 \mathrm{~mm}$ cord from the center for OK treatment and in the $4 \mathrm{~mm}$ cord from the center for the LASIK group. These tables further evidences a statistically significant increase in mid-peripheral corneal power $(P<0.001)$ measured as local steepening in the 6-mm cord for both OK and LASIK treatments. Simultaneously, there was a statistically significant central corneal thinning $(P$

TABLE 2. Descriptive Statistics (Mean \pm SD) of the Difference between Corneal Topography and Thickness Pre- and Post-treatment over the 12-Month Period for the OK Group

\begin{tabular}{lrrrrrr}
\hline & \multicolumn{3}{c}{ Curvature (D) } & & \multicolumn{2}{c}{ Thickness $(\mu \mathrm{m})$} \\
\cline { 2 - 3 } \cline { 6 - 7 } Post-Pre (OK) & Mean \pm SD & $\boldsymbol{P}^{*}$ & & Mean \pm SD & $\boldsymbol{P}^{*}$ \\
\hline Center & $-2.59 \pm 1.06$ & $<0.001$ & & $-22.25 \pm 12.13$ & $<0.001$ \\
Cord $2 \mathrm{~mm}$ & $-1.45 \pm 1.19$ & $<0.001$ & & $-20.34 \pm 9.95$ & $<0.001$ \\
Cord $4 \mathrm{~mm}$ & $+0.55 \pm 1.92$ & 0.117 & $-11.53 \pm 15.19$ & $<0.001$ \\
Cord 6 mm & $+1.40 \pm 1.99$ & $<0.001$ & & $+17.18 \pm 18.23$ & $<0.001$ \\
\hline
\end{tabular}

\footnotetext{
* Paired sample $t$-test.
}

$<0.001)$ in the $4-\mathrm{mm}$ cord from the center, and a midperipheral (6-mm cord) thickening over the 12-month period for the OK subjects.

\section{Inflammatory Mediators}

Levels of inflammatory mediators and significance after $\mathrm{OK}$ and LASIK treatments compared with controls are shown in Table 4. Median values of IL-6 and IL-8 increased significantly $(P<$ 0.001 ) in the tear samples collected from the OK lens group in comparison with those obtained from the control or LASIK subjects. Mann-Whitney test confirmed that EGF and MMP-9 ( $P$ $<0.001)$ concentrations were significantly greater in the tears collected from both, the LASIK $(P=0.002$ and $P<0.001$ respectively) and the OK $(P<0.001)$ groups as per comparison with the control subjects.

The levels of inflammatory markers in tears assayed for the OK subjects $(P<0.001)$, and the MMP-9 $(P=0.001)$ and EGF $(P<0.001)$ mean tear levels for the LASIK group were significantly associated with the degree of myopia correction. Values measured after treatment with contact lenses and LASIK in comparison with controls can be graphically observed in Figures 1 and 2.

A very interesting clinical finding of this study was the presence of deposition, presumably of iron (Fig. 3), in 60\% of the patients undergoing OK treatment for 12 months, and only in $10 \%$ of the patients that underwent LASIK. When the magnitude of the regulatory response was analyzed in relation to the presence of iron deposition, MMP-9 was found to have increased in eyes presenting iron ring compared with treated eyes, without iron deposition, in OK $(P=0.001$; Fig. 4) and LASIK ( $P=0.019$, Fig. 5$)$ groups. For the OK patients, EGF was

TABLE 3. Descriptive Statistics (Mean \pm SD) of the Difference between Corneal Topography and Thickness Pre- and Post-treatment over the 12-Month Period for the LASIK Group

\begin{tabular}{|c|c|c|c|c|}
\hline \multirow[b]{2}{*}{ Post-Pre (LASIK) } & \multicolumn{2}{|c|}{ Curvature (D) } & \multicolumn{2}{|c|}{ Thickness $(\mu \mathrm{m})$} \\
\hline & Mean \pm SD & $\boldsymbol{P}^{*}$ & Mean $\pm S D$ & $\boldsymbol{P}^{*}$ \\
\hline Center & $-2.83 \pm 1.02$ & $<0.001$ & $-45.30 \pm 26.13$ & $<0.001$ \\
\hline Cord $2 \mathrm{~mm}$ & $-2.33 \pm 1.20$ & $<0.001$ & $-38.67 \pm 25.40$ & $<0.001$ \\
\hline Cord $4 \mathrm{~mm}$ & $-0.96 \pm 1.06$ & $<0.001$ & $-22.47 \pm 18.71$ & $<0.001$ \\
\hline Cord $6 \mathrm{~mm}$ & $+1.37 \pm 1.04$ & $<0.001$ & $-4.90 \pm 10.19$ & 0.013 \\
\hline
\end{tabular}

\footnotetext{
* Paired sample $t$-test.
} 
TABLE 4. Descriptive Analysis and Significance for IL-6, IL-8, EGF, and MMP-9 Data Sets in the Tear Samples Collected from OK and LASIK Groups Compared with Control Values

\begin{tabular}{|c|c|c|c|c|c|}
\hline \multirow[b]{2}{*}{ Case-Control } & \multirow{2}{*}{$\begin{array}{c}\text { Control }(n=32) \\
\text { Median [Range] }\end{array}$} & \multicolumn{2}{|c|}{ OK $(n=32)$} & \multicolumn{2}{|c|}{ LASIK $(n=30)$} \\
\hline & & Median [Range] & $\boldsymbol{P}^{*}$ & Median [Range] & $\boldsymbol{P}^{*}$ \\
\hline IL-6, pg/mL & $2.2[1.4-3.6]$ & $4.7[3.8-8.6]$ & $<0.001$ & $2.5[2.0-5.8]$ & 0.056 \\
\hline IL-8, pg/mL & $601.5[477.1-745.5]$ & $935.3[657.5-1502.5]$ & $<0.001$ & 624.6 [505.2-1241.6] & 0.081 \\
\hline MMP-9, ng/mL & $39.2[32.8-61.2]$ & $74.3[54.9-112.3]$ & $<0.001$ & $51.1[42.8-83.3]$ & $<0.001$ \\
\hline EGF, pg/mL & 698.1 [463.8-1390.4] & 2348.7 [1594.9-4135.4] & $<0.001$ & 990.2 [564.7-1739.4] & 0.002 \\
\hline
\end{tabular}

* Mann-Whitney test

also found to have increased significantly $(P=0.039$; Fig. 4). Tables 5 and 6 show the mean difference, SD, and significance between levels of mediators in tears after OK and LASIK treatments in comparison with controls.

Finally, a myopic regression was observed in 7 cases (23\%) with a loss of 1 or 2 Snellen VA lines 12 months after LASIK surgery, with a mean spherical equivalent refraction of -0.77 $\pm 0.32 \mathrm{D}$. The Mann-Whitney test shows that only a significant increase in the EGF levels $(P=0.001)$ can be observed in
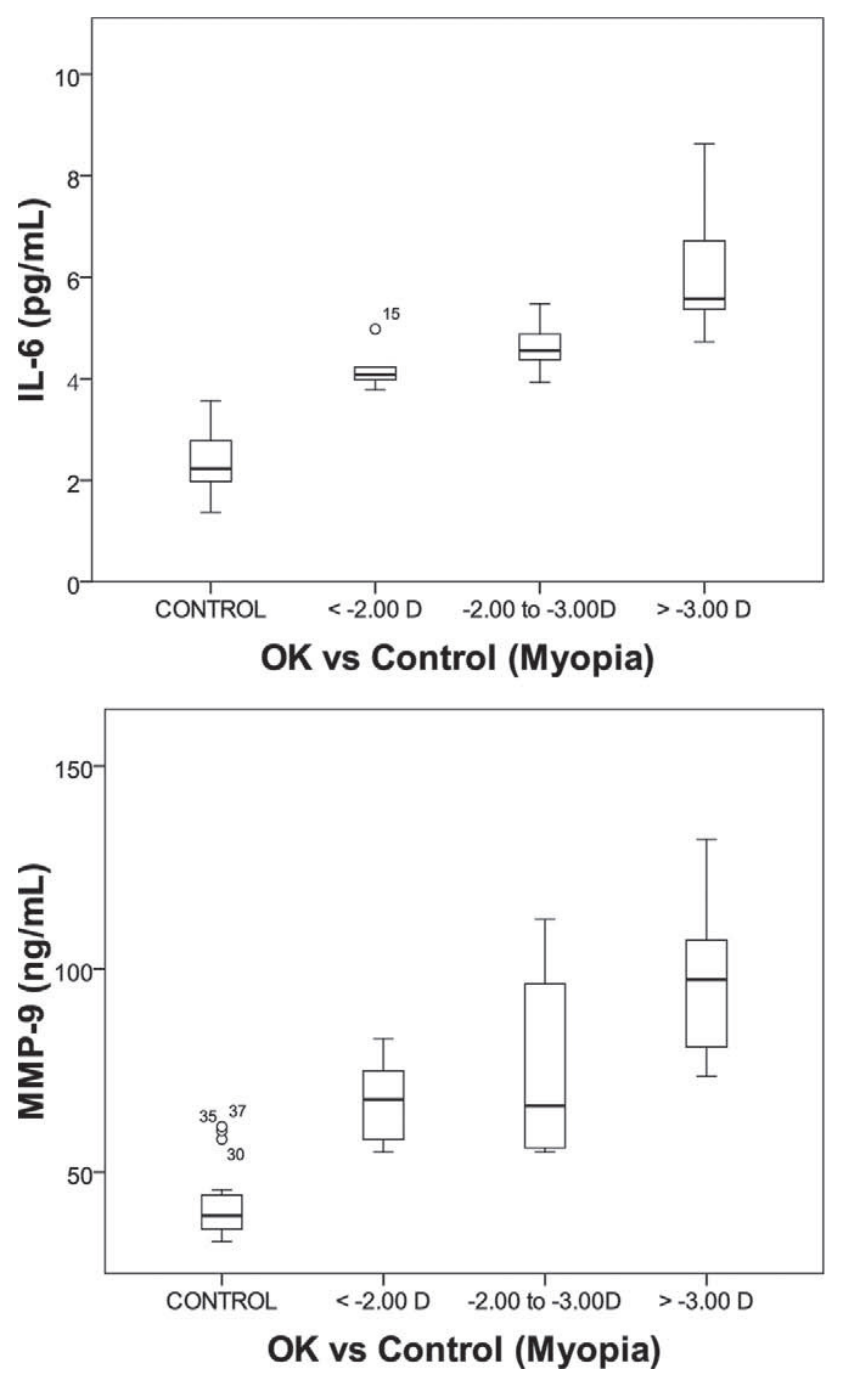

connection with the myopic regression effect that occurred 12 months after surgery (Fig. 6).

\section{Discussion}

To the best of the authors' knowledge, there are no studies involving the evaluation of inflammatory mediators in tears of OK lens wearers compared with controls or LASIK patients. There are, however, several studies involving the short-term
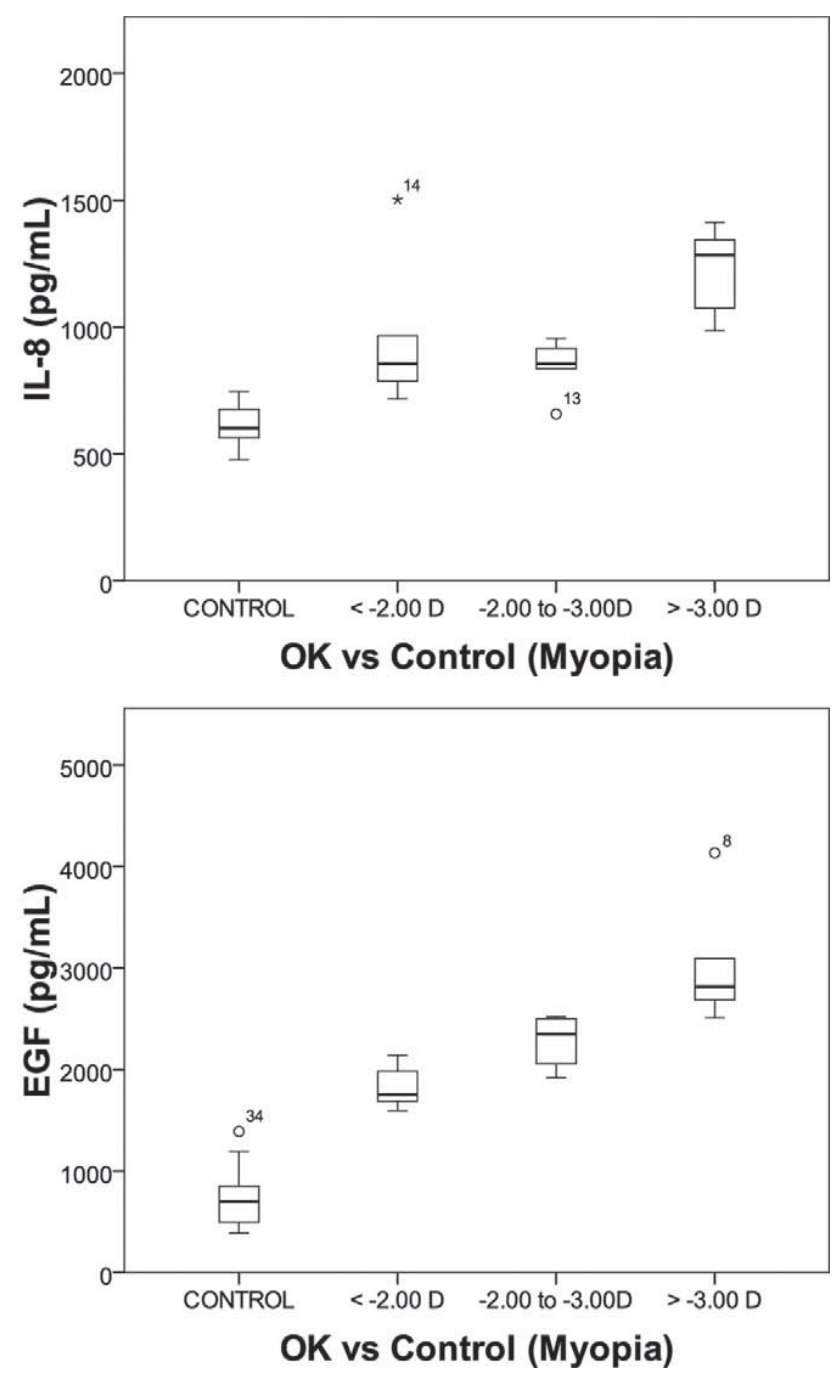

FIGURE 1. Box plots representing IL-6, IL-8, MMP-9, and EGF concentrations in tear samples collected from the control subjects and the OK group. A significant increase in the levels of all inflammatory mediators $(P<0.001)$ can be observed in association with the amount of treated myopia for OK lens wearers. 

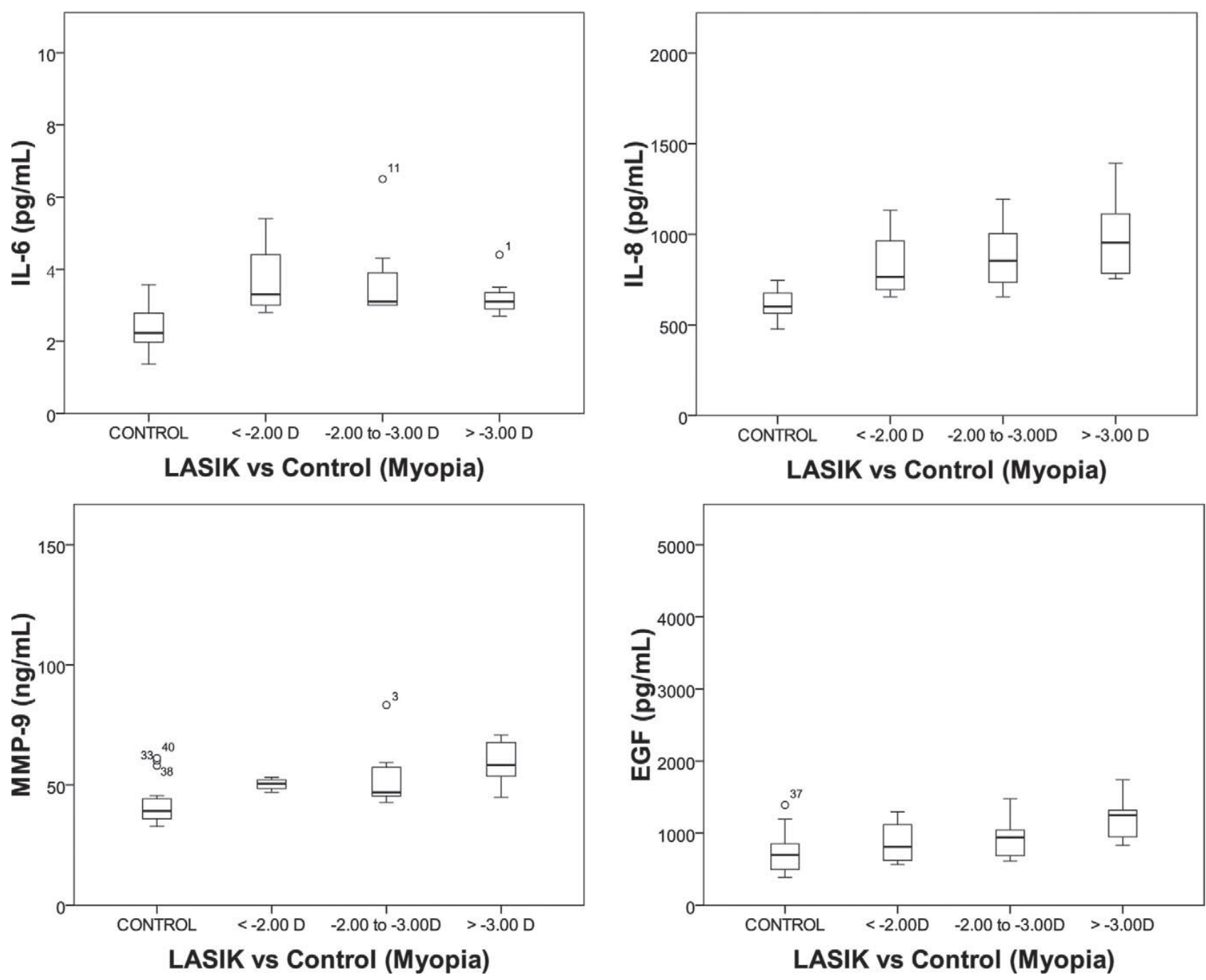

FIGURE 2. Box plots representing IL-6, IL-8, MMP-9, and EGF concentrations in tear samples collected from the control subjects and LASIK patients Only a significant increase in the levels of MMP-9 and EGF $(P<0.05)$ can be observed in association with the amount of treated myopia for LASIK subjects.

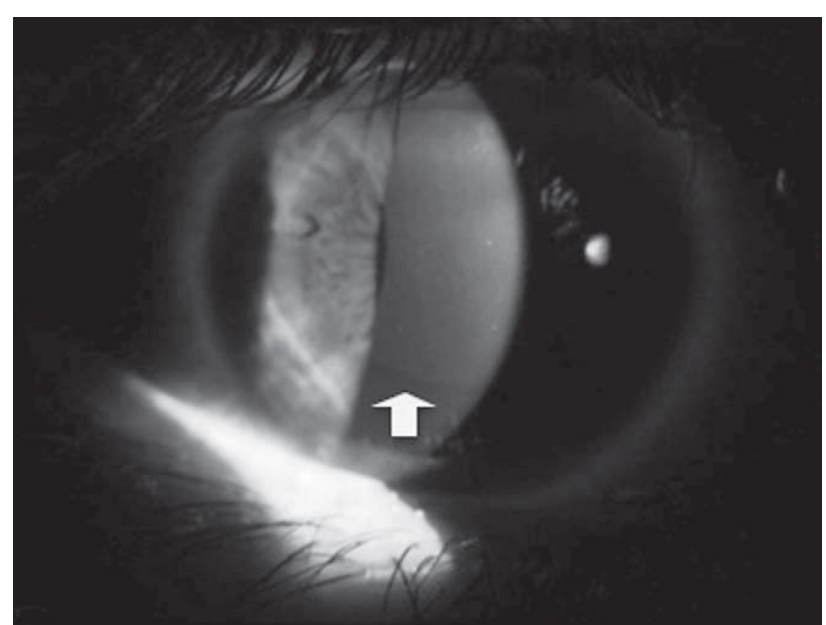

FIgURE 3. Presumed iron ring during OK therapy in the midperipheral cornea. inflammatory response after photorefractive keratectomy (PRK) or LASIK, indicating that different techniques stimulate cytokines, chemokines, and growth factors, which are known to regulate wound healing, apoptosis, cell homeostasis, and migration of the cornea in the early period after surgery. However, the pattern of cytokine, matrix protease, or growth factor release in the long-term period after LASIK is not well delineated.

In the present investigation, we used the ELISA method to measure a pattern of cytokine, chemokine, matrix protease, and growth factor production in tears after OK and LASIK treatments. This study has demonstrated increased levels of pro-inflammatory markers, matrix proteinase, and EGF in the tears of subjects wearing OK contact lenses over a 12-month period. The magnitude of this response was found to be associated with the degree of myopia, suggesting the OK compressive effect and mechanical trauma as the main causes of the inflammatory upregulation. This effect may be associated with the remarkable changes in corneal structure (corneal curvature and thickness) induced by the OK treatment. ${ }^{3,15,19}$ Conversely, there is not a long-term proinflammatory effect after LASIK, although increased levels of 

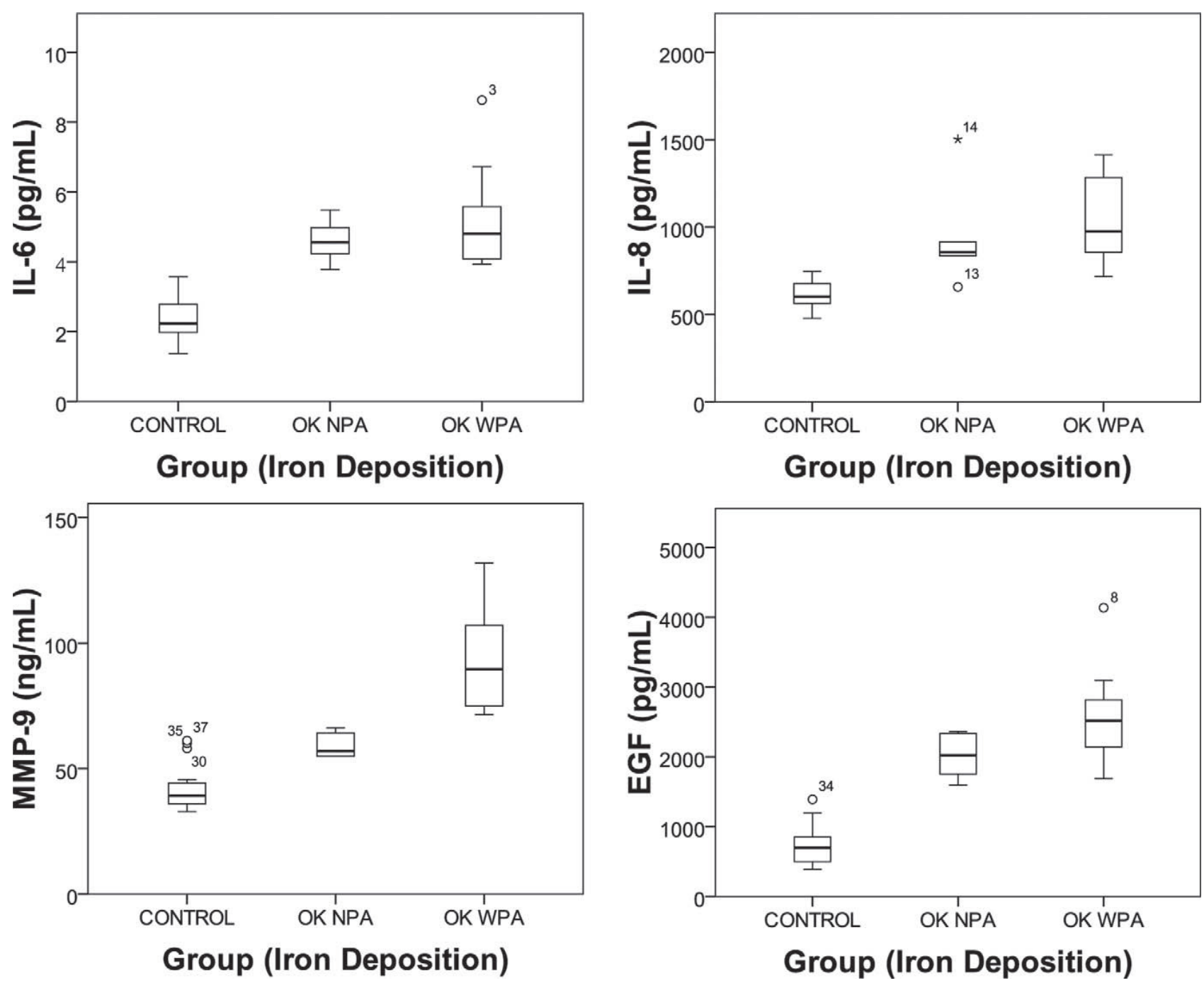

FIGURE 4. Box plots representing IL-6, IL-8, MMP-9, and EGF concentrations in tear samples collected from the control subjects and the OK group. A significant increase in the levels of MMP-9 $(P=0.001)$ and EGF $(P=0.039)$ can be observed in association with the presence of iron deposition in the corneal epithelium for OK subjects. NPA, no pigmented arc; WPA, with pigmented arc.

matrix proteinase and EGF were found to be associated with different clinical outcomes, such as iron deposition and myopic regression.

Evaluation of structural changes after treatments show different alterations in corneal thickness and in the power of the anterior corneal surface from the center toward the peripheral cornea, which is due to different corneal refractive treatment options (surgical and nonsurgical) for the correction of myopia. The tangential power of the anterior corneal surface after the refractive treatments showed, as expected, a loss of central corneal power, whereas in the mid-peripheral zone (6 $\mathrm{mm}$ ) there was an increase in power. This occurred differently in each treatment. At the paracentral area beyond the central 2 $\mathrm{mm}$, corneal power decreased after LASIK treatments and remained unchanged or increased slightly after the OK treatment. Simultaneously, the central cornea thins, whereas the mid-periphery thickens as a result of the notable tissue redistribution described previously for the OK group, ${ }^{3,15}$ with no changes for the LASIK subjects. In this study, we detected in the OK group an initial spherical equivalent refractive error of $-2.69 \pm 1.07 \mathrm{D}$ and a reduction of $-2.59 \pm 1.06 \mathrm{D}$ in the central power of the cornea (with a thinning effect of -22.25 $\pm 12.13 \mu \mathrm{m}$ ), with a rise of $+1.40 \pm 1.99 \mathrm{D}$ (with a thickening effect of $+17.18 \pm 18.23 \mu \mathrm{m}$ ) in the mid-peripheral zone after 12 months of treatment. In turn, the LASIK group presented an initial spherical equivalent refractive error $-2.99 \pm 1.03 \mathrm{D}$ and a reduction of $-2.83 \pm 1.02 \mathrm{D}$ in the central power of the cornea (with a stromal subtraction of $-45.30 \pm 26.13 \mu \mathrm{m}$ ), with a rise of $+1.37 \pm 1.04 \mathrm{D}$ (with a paradoxal thinning effect of $-4.90 \pm 10.19 \mu \mathrm{m}$ ) in the mid-peripheral zone after 12 months of treatment. Similar results to those found for OK and LASIK in this study had been reported by other authors. ${ }^{15,20,21}$

It is possible that overexpression of chemokines and other molecules involved in the wound-healing process are responsible for noninfective post-LASIK or post-OK complications including different nonsymptomatic clinical outcomes. Most studies report a short-term upregulation of several cytokines and chemokines in the corneal wound-healing process after LASIK that might initiate potentially severe corneal inflammation, leading to corneal haze and other unsatisfactory sequelae. ${ }^{14,22-26}$ Contact lens-induced trauma to the epithelium could result in increased release of inflammatory media- 

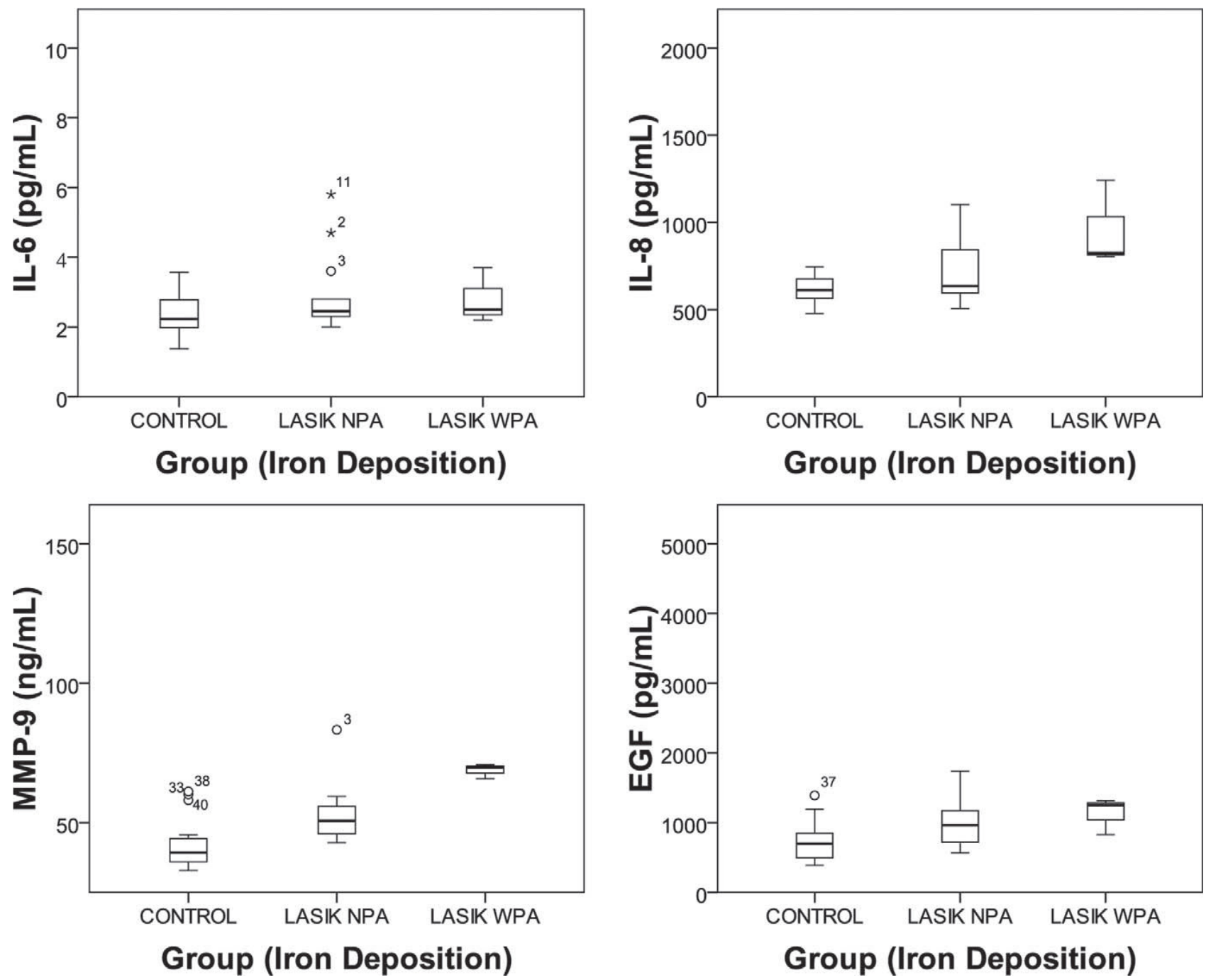

FIGURE 5. Box plots representing IL-6, IL-8, MMP-9, and EGF concentrations in tear samples collected from the control subjects and LASIK group. A significant increase in the levels of MMP-9 $(P=0.019)$ can be observed in association with the presence of iron deposition in the corneal epithelium for the LASIK group. NPA, no pigmented arc; WPA, with pigmented arc.

tors, facilitating the loss of corneal keratocytes and long-term stromal thinning. Previous reports of inflammatory mediators in tears of contact lens wearers have shown the altered presence of histamine, granulocyte macrophage colony-stimulating factor, TNF- $\alpha$, IL-6, IL-8, leukotriene B4, MMP-9, and $\mathrm{EGF}^{8-11}$

In this study, tear IL-6, although at moderate levels, increased 12 months after the OK treatment in almost all subjects $(P<0.05)$; however, as expected, it was not elevated in the long-term period after LASIK probably due to a lack of keratocyte stimulation in the 12 months after surgery. IL-6 is a multipotent pro-inflammatory cytokine synthesized by keratocytes and endothelial cells in response to pro-inflammatory stimuli. IL-6 stimulates epithelial migration in the cornea ${ }^{27,28}$ and can also induce the expression of MMPs. ${ }^{29}$ IL- 6 is a constant component of the normal human tear fluid ${ }^{6}$ and has

TABLE 5. Descriptive Statistics of the Difference between Levels of Mediators in Tears after Treatment Compared with Controls (Mean \pm SD) for OK

\begin{tabular}{|c|c|c|c|c|c|c|}
\hline \multirow[b]{2}{*}{ OK } & \multicolumn{2}{|c|}{ NPA (12) vs. Control (32) } & \multicolumn{2}{|c|}{ WPA (20) vs. Control (32) } & \multicolumn{2}{|c|}{ WPA (20) vs. NPA (12) } \\
\hline & Mean \pm SD & $\boldsymbol{P}^{*}$ & Mean \pm SD & $\boldsymbol{P}^{k}$ & Mean \pm SD & $\boldsymbol{P}^{k}$ \\
\hline IL-6, pg/mL & $+2.30 \pm 0.26$ & $<0.001$ & $+2.93 \pm 0.36$ & $<0.001$ & $+0.63 \pm 0.64$ & 0.515 \\
\hline IL-8, pg/mL & $+323.26 \pm 66.57$ & 0.001 & $+423.98 \pm 55.47$ & $<0.001$ & $+100.72 \pm 133.27$ & 0.278 \\
\hline MMP-9, $\mathrm{ng} / \mathrm{mL}$ & $+17.06 \pm 3.63$ & 0.002 & $+50.89 \pm 5.02$ & $<0.001$ & $+33.82 \pm 8.35$ & 0.001 \\
\hline EGF, pg/mL & $+1312.16 \pm 131.20$ & $<0.001$ & $+1898.72 \pm 170.70$ & $<0.001$ & $+586.56 \pm 297.87$ & 0.039 \\
\hline
\end{tabular}

NPA, no pigmented arc; WPA, with pigmented arc

* Mann-Whitney test. 
TABLE 6. Descriptive Statistics of the Difference between Levels of Mediators in Tears after Treatment Compared with Controls (Mean \pm SD) for LASIK

\begin{tabular}{|c|c|c|c|c|c|c|}
\hline \multirow[b]{2}{*}{ LASIK } & \multicolumn{2}{|c|}{ NPA (27) vs. Control (32) } & \multicolumn{2}{|c|}{ WPA (3) vs. Control (32) } & \multicolumn{2}{|c|}{ WPA (3) vs. NPA (27) } \\
\hline & Mean \pm SD & $\boldsymbol{P}^{*}$ & Mean \pm SD & $\boldsymbol{P}^{*}$ & Mean \pm SD & $\boldsymbol{P}^{*}$ \\
\hline IL-6, pg/mL & $+0.53 \pm 0.26$ & 0.051 & $+0.50 \pm 0.35$ & 0.209 & $-0.03 \pm 0.64$ & 0.866 \\
\hline IL-8, pg/mL & $+91.97 \pm 43.17$ & 0.193 & $+334.16 \pm 61.85$ & 0.006 & $+242.20 \pm 121.48$ & 0.083 \\
\hline MMP-9, ng/mL & $+10.64 \pm 2.93$ & 0.012 & $+27.05 \pm 4.91$ & 0.006 & $+16.40 \pm 5.76$ & 0.019 \\
\hline EGF, pg/mL & $+284.43 \pm 97.81$ & 0.005 & $+423.27 \pm 166.73$ & 0.045 & $+138.83 \pm 203.63$ & 0.502 \\
\hline
\end{tabular}

* Mann-Whitney test.

been found to be upregulated in different active ocular surface inflammatory conditions or after corneal epithelial aggression, including RGP lens wear. ${ }^{9,13,30,31}$ In this work, $\mathrm{a} \times 2.1$ increase was found in tear samples of OK lens wearers, which is in agreement with previous studies using RGP lenses.9,13 Nevertheless, we found a stronger effect that might be explained by the epithelial reshaping effect of the reverse geometry $\mathrm{OK}$ lenses and lens induced-hypoxia. In that context, IL-6 may act as a stimulating factor in the epithelial
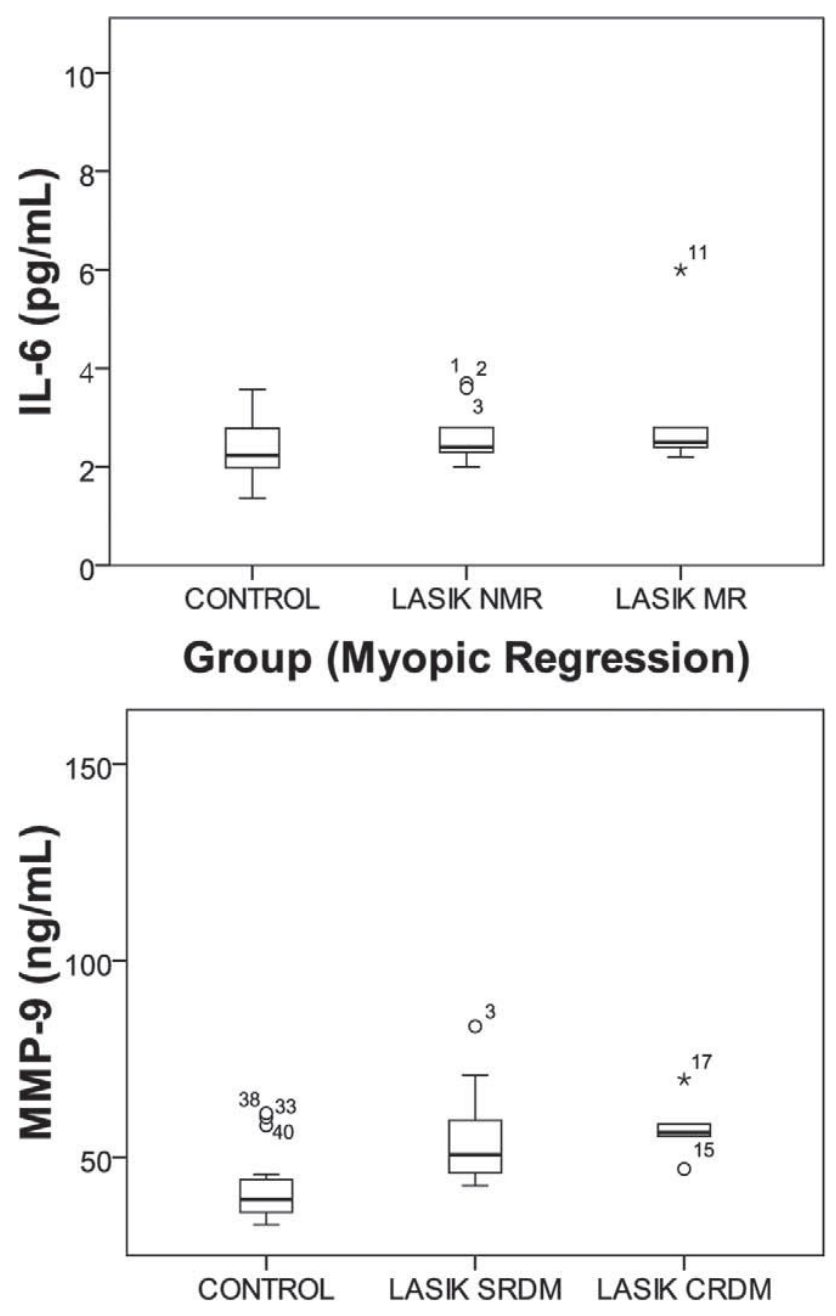

Group (Myopic Regression) cell migration, probably by means of a fibronectin-dependent mechanism, ${ }^{27,28}$ from the central cornea toward the midperiphery during the $\mathrm{OK}$ tissue redistribution process. ${ }^{3,32}$

The other cytokine analyzed in this work was IL-8. A potent neutrophil chemotactic and activating factor were also present in tears from controls and found to be significantly higher in the samples of OK lens wearers when compared with those of LASIK patients and control subjects. This finding is consistent with the results of previous studies using disposable and rigid
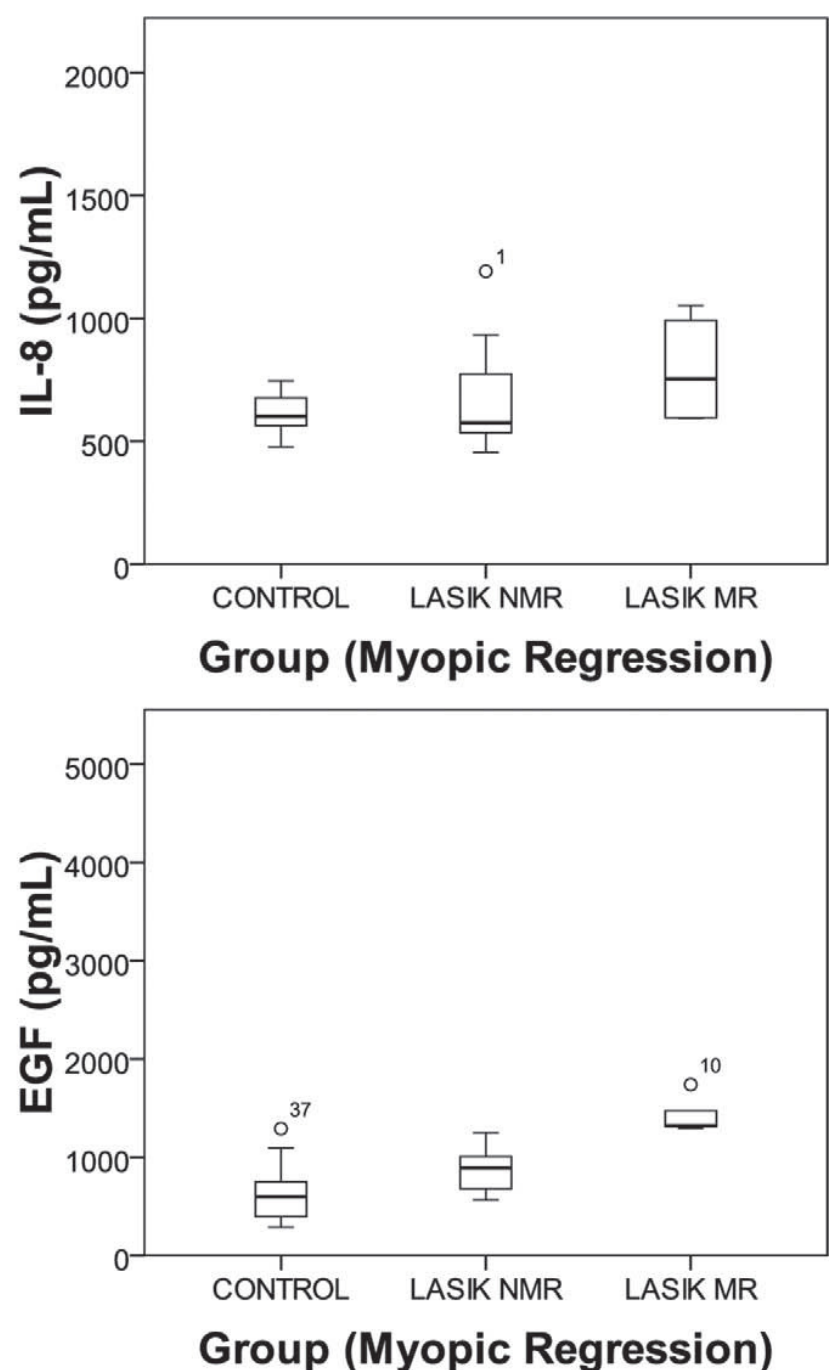

Figure 6. Box plots representing IL-6, IL-8, MMP-9, and EGF concentrations in tear samples collected from the control subjects and the LASIK group. A significant increase in the levels of EGF $(P=0.001)$ can be observed in association with the presence of myopic regression (MR) for LASIK patients. NMR, no myopic regression. 
contact lenses worn overnight. ${ }^{8,11}$ In our study, the major elevation of this cytokine seems to occur due to the persistent and remarkable mechanical effect of OK lenses and also probably due to a lens induced-hypoxia effect, which are held as the main causes of this subclinical pro-inflammatory effect.

MMP-9 is the primary matrix-degrading enzyme produced by the corneal epithelium and fibroblasts in response to proinflammatory stimuli such as IL-1 and TNF- $\alpha .{ }^{33}$ There is evidence to suggest that MMPs play a vital role in several physiological and pathological processes. They participate in extracellular matrix remodeling after wounding of the corneal surface and MMP-9 has proved crucial in catalyzing the cleavage of epithelial basement membrane components. ${ }^{34}$ In the present study, we have found that, for subjects wearing $\mathrm{OK}$ lenses, the concentration of MMP-9 was elevated by a factor of 1.9 in a similar proportion to that of IL-6 cytokine. In this study, we present for the first time the MMP-9 inducing extracellular matrix degradation as a facilitating mechanism involved in the corneal molding process and the maintenance of the OK effect. Our study further revealed that tears from LASIK patients also have significantly increased levels of MMP- $9(\times 1.3)$ compared with controls, suggesting that the extracellular matrix remodeling process may continue up to 12 months after surgery, especially for subjects who have had a higher tissue removal.

Another possible effect derived from MMP-9 upregulation is related to the long-term pigment deposition at the basement epithelium after surgical and nonsurgical treatments. Corneal iron deposition was present in $60 \%$ of the cases of overnight OK lens wear in this sample of patients, and in $10 \%$ of the patients who have undergone LASIK. This finding is in agreement with previous literature and was more prominent in patients with higher baseline refractive errors. ${ }^{35-37}$ Consistent with other types of pigment lines found in keratoconus patients, the pigmentation does not interfere with the visual axis and does not alter visual acuity in these subjects; hence, no treatment was required ${ }^{38}$ Although corneal iron deposits in various patterns have been reported following other forms of ocular therapeutic and refractive surgery, this is the first report on the association between increased levels of MMP-9 or EGF and corneal iron line following LASIK or OK treatments. This iron deposition within the margin of the ablated zone for LASIK patients or in the mid-peripheral cornea in relation to the landing zone for the OK subjects may offer insights into the dynamics of epithelial cell hyperplasia, as well as basal cell proliferation, differentiation, and migration after OK or LASIK, which partly explains the increased rate of EGF found in this investigation. Moreover, given the role of MMP-9 in the management of epithelial basal membrane compounds, the increased levels of MMP-9 in eyes showing iron ring pigmentation might be related to an increased rate of extracellular matrix degradation causing iron and other deposition in the epithelium. Considering the limited sample size of patients with iron deposition in the LASIK group, these results must be considered only as trends and no definitive conclusions should be drawn.

Tear EGF increased to high levels after OK (especially for more myopic subjects), and to moderate levels after LASIK. EGF is a mitogenic protein involved in several mechanisms, such as normal cell growth, oncogenesis, and wound healing. It has been proved to be a constant component of normal tears and has been found to be upregulated after epithelial injury or contact lens wear. ${ }^{8,39,40}$ In our study, EGF showed the major upregulation in the OK group, with an increase of $\times 3.4$ for the OK lens wearers. Although there are no previous studies with OK lenses, this finding is consistent with the results of previous studies using RGP lenses, which report an increase of $\times 2.4$ in EGF levels due to the contact lens-induced mechanical effect. $^{8}$ The EGF overexpression in the OK lens-wearing group reported in this research probably occurs in response to the potent mechanical effect of reverse geometry rigid contact lenses, inducing a short-term corneal epithelium redistribution and a long-term overnight structural maintenance for the duration of the treatment. 3,32 Differently, this study shows an EGF increase of $\times 1.4,12$ months after LASIK. Using the ELISA method, several researchers have reported increased EGF levels in the wound-healing response after surgery associated with stromal regrowth and epithelial hyperplasia, which are phenomena involved in refractive instability after refractive surgery. ${ }^{41-44}$ Ivarsen et al. ${ }^{44,45}$ found a progressive epithelial hyperplasia 12 months after PRK and 2 months after LASIK. Lohmann et al. ${ }^{42,43}$ reported an increased epithelial woundhealing response with increased EGF levels, associated with postoperative refractive outcome several months after PRK and myopic LASIK. These authors suggested that therapeutic control of EGF should be considered for the possibility of myopic regression control. Data found in the aforementioned study is in agreement with the present study, which provides a possible mechanism for this regression and thus supports the possibility of pharmaceutical manipulation.

A possible source of error in this study concerning the obtainment of concentration values of tear markers could be the alteration of tear production after LASIK or during OK treatments. Tear secretion is typically decreased in the first month after LASIK and resolve in the vast majority of patients, but there is considerable variability between individual eyes. ${ }^{46,47}$ Although the mechanisms for developing post-LASIK dry eye are not completely understood, loss of corneal innervation by flap-making may affect the reflex loops of the corneal-lacrimal gland, corneal-blinking, and blinking-meibomian gland, resulting in decreased aqueous and lipid tear secretion and mucin expression and finally causing discomfort in some patients. Tear quality/quantity reduction and corneal staining occurred in many subjects during the OK procedure, mainly in subjects with tear deficiencies. ${ }^{48,49}$ Recent research also supports the changes in the sub-basal neural plexus during the OK treatment. ${ }^{50}$ The mechanical effect of contact lenses due to inverse geometry design has been suggested as the main cause. Although these factors might affect the tear production after LASIK and OK treatments, we only considered asymptomatic those subjects with tear characteristics within normal limits, as defined in the inclusion criteria, in order to minimize any potential effect on the consistency of concentration measures of tear markers.

In conclusion, the surgical and nonsurgical alternatives for myopic correction analyzed in this study induce different levels of long-term subclinical inflammation and this condition is more significant with overnight $\mathrm{OK}$ treatment than with LASIK. The persistent nature of $\mathrm{OK}$ and the proportional increase in this upregulation with the level of myopia being corrected in OK corroborates the hypothesis that links this response to the physical impact of contact lenses on the corneal epithelium during treatment. In addition, overexpression of cytokines and other molecules involved in the woundhealing process are associated with the long-term post-LASIK or post-OK clinical outcomes, including nonsymptomatic iron deposition and myopic regression. Thus, the role of cytokines, chemokines, matrix proteases, and growth factors in the longterm postoperative LASIK period and during OK must be further evaluated.

\section{Acknowledgments}

The authors thank Isabel Lema, MD, PhD, for assistance with tear sample collection and data interpretation, as well as Tomás Sobrino, PhD, for assistance with tear marker assays and Francisco Avilés, OD, for help with data management. 


\section{References}

1. Sorbara L, Fonn D, Simpson T, Lu F, Kort R. Reduction of myopia from corneal refractive therapy. Optom Vis Sci. 2005; 82:512-518

2. Walline JJ, Jones LA, Sinnott LT. Corneal reshaping and myopia progression. Br J Ophthalmol. 2009;93:1181-1185.

3. Alharbi A, Swarbrick HA. The effects of overnight orthokeratology lens wear on corneal thickness. Invest Opbthalmol Vis Sci. 2003;44:2518-2523.

4. Alió JL, Pérez-Santonja JJ, Tervo T, et al. Postoperative inflammation, microbial complications, and wound healing following laser in situ keratomileusis. J Refract Surg. 2000;16: 523-538.

5. Lopez JG, Chew SJ, Thompson HW, et al. EGF cell surface receptor quantitation on ocular cells by an immunocytochemical flow cytometry technique. Invest Ophthalmol Vis Sci. 1992;33:2053-2062.

6. Nakamura Y, Sotozono C, Kinoshita S. Inflammatory cytokines in normal human tears. Curr Eye Res. 1998;17:673-676.

7. Sack RA, Conradi L, Krumholz D, Beaton A, Sathe S, Morris C. Membrane array characterization of 80 chemokines, cytokines, and growth factors in open- and closed-eye tears: angiogenin and other defense system constituents. Invest Ophthalmol Vis Sci. 2005;46:1228-1238.

8. Kallinikos P, Morgan P, Efron N. Assessment of stromal keratocytes and tear film inflammatory mediators during extended wear of contact lenses. Cornea. 2006;25:1-10.

9. Lema I, Durán JA, Ruiz C, Díez-Feijoo E, Acera A, Merayo J. Inflammatory response to contact lenses in patients with keratoconus compared with myopic subjects. Cornea. 2008; 27:758-763.

10. Sack RA, Sathe S, Beaton A. Tear turnover and immune and inflammatory processes in the open-eye and closed-eye environments: relationship to extended wear contact lens use. Eye Contact Lens. 2003;29:S80-S82.

11. Thakur A, Willcox MD. Contact lens wear alters the production of certain inflammatory mediators in tears. Exp Eye Res. 2000;70:255-259.

12. Vesaluoma M, Tervo T. Tear fluid changes after photorefractive keratectomy. Adv Exp Med Biol. 1998;438:515-521.

13. Leonardi A, Curnow SJ, Zhan H, Calder VL. Multiple cytokines in human tear specimens in seasonal and chronic allergic eye disease and in conjunctival fibroblast cultures. Clin Exp Allergy. 2006;36:777-784.

14. Leonardi A, Tavolato M, Curnow SJ, Fregona IA, Violato D, Alió JL. Cytokine and chemokine levels in tears and in corneal fibroblast cultures before and after excimer laser treatment. $J$ Cataract Refract Surg. 2009;35:240-247.

15. Lu F, Simpson T, Sorbara L, Fonn D. The relationship between the treatment zone diameter and visual, optical and subjective performance in Corneal Refractive Therapy lens wearers. Ophthalmic Physiol Opt. 2007;27:568-578.

16. Holladay JT, Dudeja DR, Chang J. Functional vision and corneal changes after laser in situ keratomileusis determined by contrast sensitivity, glare testing, and corneal topography. $J$ Cataract Refract Surg. 1999;25:663-669.

17. Gonzalez-Meijome JM, Villa-Collar C. Nomogram, corneal topography, and final prescription relations for corneal refractive therapy. Optom Vis Sci. 2007;84:59-64.

18. Handa T, Mukuno K, Niida T, Uozato H, Tanaka S, Shimizu K. Diurnal variation of human corneal curvature in young adults. J Refract Surg. 2002;18:58-62.

19. Haque S, Fonn D, Simpson T, Jones L. Corneal and epithelial thickness changes after 4 weeks of overnight corneal refractive therapy lens wear, measured with optical coherence tomography. Eye Contact Lens. 2004;30:189-193.
20. Hashemi H, Mehravaran S. Corneal changes after laser refractive surgery for myopia: comparison of Orbscan II and Pentacam findings. J Cataract Refract Surg. 2007;33:841-847.

21. Queiros A, Gonzalez-Meijome JM, Villa-Collar C, et al. Local steepening in peripheral corneal curvature after corneal refractive therapy and LASIK. Optom Vis Sci. 2010;87:432439.

22. Tran MT, Tellaetxe-Isusi M, Elner V, Strieter RM, Lausch RN, Oakes JE. Proinflammatory cytokines induce RANTES and MCP-1 synthesis in human corneal keratocytes but not in corneal epithelial cells; $\beta$-chemokine synthesis in corneal cells. Invest Ophthalmol Vis Sci. 1996;37:987-996.

23. Hong JW, Liu JJ, Lee JS, et al. Proinflammatory chemokine induction in keratocytes and inflammatory cell infiltration into the cornea. Invest Ophthalmol Vis Sci. 2001;42:2795-2803.

24. McInnis KA, Britain A, Lausch RN, Oakes JE. Synthesis of $\alpha$ chemokines IP-10, I-TAC, and MIG are differentially regulated in human corneal keratocytes. Invest Ophthalmol Vis Sci. 2005; 46 :1668-1674.

25. Wilson SE, Mohan RR, Mohan RR, Ambrosio R, Hong J, Lee J. The corneal wound healing response: cytokine-mediated interaction of the epithelium, stroma, and inflammatory cells. Prog Retin Eye Res. 2001;20:625-637.

26. Wilson SE, Mohan RR, Hong JW, Lee JS, Choi R, Mohan RR. The wound healing response after laser in situ keratomileusis and photorefractive keratectomy; elusive control of biological variability and effect on custom laser vision correction. Arch Opbthalmol. 2001;119:889-896.

27. Nakamura M, Nishida T. Differential effects of epidermal growth factor and interleukin 6 on corneal epithelial cells and vascular endothelial cells. Cornea. 1999;18:452-458.

28. Nishida T, Nakamura M, Mishima H, Otori T. Interleukin 6 promotes epithelial migration by a fibronectin-dependent mechanism. J Cell Pbysiol. 1992;153:1-5.

29. Girolamo ND, Kumar RK, Coroneo MT, Wakefield D. UVBmediated induction of interleukin- 6 and -8 in pterygia and cultured human pterygium epithelial cells. Invest Ophthalmol Vis Sci. 2002; 43:3430-3437.

30. Schultz CL, Kunert KS. Interleukin-6 levels in tears of contact lens wearers. J Interferon Cytokine Res. 2000;20:309-310.

31. Yang SF, Lin CY, Yang PY, Chao SC, Ye YZ, Hu DN. Increased expression of gelatinase (MMP-2 and MMP-9) in pterygia and pterygium fibroblasts with disease progression and activation of protein kinase C. Invest Ophthalmol Vis Sci. 2009;50:45884596.

32. Choo JD, Caroline PJ, Harlin DD, Papas EB, Holden BA. Morphologic changes in cat epithelium following continuous wear of orthokeratology lenses: a pilot study. Cont Lens Anterior Eye. 2008;31:29-37.

33. Gordon GM, Ledee DR, Feuer WJ, Fini ME. Cytokines and signaling pathways regulating matrix metalloproteinase-9 (MMP-9) expression in corneal epithelial cells. J Cell Pbysiol. 2009;221:402-411.

34. Fukuda K, Fujitsu Y, Kumagai N, Nishida T. Inhibition of matrix metalloproteinase-3 synthesis in human conjunctival fibroblasts by interleukin- 4 or interleukin-13. Invest Ophthalmol Vis Sci. 2006; 47:2857-2864.

35. Liang JB, Chou PI, Wu R, Lee YM. Corneal iron ring associated with orthokeratology. J Cataract Refract Surg. 2003;29:624626.

36. Cheung SW, Cho P, Cheung A. White lesion in the corneal pigmented ring associated with orthokeratology. Ophthalmic Physiol Opt. 2005;25:264-268.

37. Vongthongsri A, Chuck RS, Pepose JS. Corneal iron deposits after laser in situ keratomileusis. Am J Opbthalmol. 1999;1: 85-86. 
38. Loh A, Hadziahmetovic M, Dunaief JL. Iron homeostasis and eye disease. Biochimica et Biophysica Acta. 2009;1790:637649.

39. Van Setten GB, Viinikka L, Tervo T, Pesonen K, Tarkkanen A, Perheentupa J. Epidermal growth factor is a constant component of normal human tear fluid. Graefes Arch Clin Exp Opbthalmol. 1989;227:184-187.

40. Wilson SE, Liang Q, Kim WJ. Lacrimal gland HGF, KGF, and EGF mRNA levels increase after corneal epithelial wounding. Invest Ophthalmol Vis Sci. 1999;40:2185-2190.

41. Chayet AS, Assil KK, Montes M, Espinosa-Lagana M, Castellanos A, Tsioulias G. Regression and its mechanisms after laser in situ keratomeileusis in moderate and high myopia. Ophthalmology. 1998;105:1194-1199.

42. Lohmann CP, Hoffmann E, Reischl U. Epidermal growth factor (EGF) in tears in excimer laser photorefractive keratectomy. Responsible for postoperative refraction and "haze"? $O p b$ thalmologe. 1998;95:80-87.

43. Lohmann CP, Von Mohrenfels CW, Reischl U, Muller A, Guell JL. Screening of myopic LASIK patients with increased epithelial wound healing. Opbthalmologe. 2001;98:460-465.
44. Ivarsen A, Mller-Pedersen T. LASIK induces minimal regrowth and no haze development in rabbit corneas. Curr Eye Res. 2005;30:363-373.

45. Ivarsen A, Fledelius W, Hjortdal J. Three-year changes in epithelial and stromal thickness after PRK or LASIK for high myopia. Invest Ophthalmol Vis Sci. 2009;50:2061-2066.

46. Shoja MR, Besharati MR. Dry eye after LASIK for myopia: incidence and risk factors. Eur J Ophthalmol. 2007;17:1-6.

47. Nettune GR, Pflugfelder SC. Post-LASIK tear dysfunction and dysesthesia. Ocul Surf. 2010;8:135-145.

48. Fan L, Jun J, Jia Q, Wangqing J, Xinjie M, Yi S. Clinical study of orthokeratology in young myopic adolescents. Int Contact Lens Clin. 1999;26:113-116.

49. Chan KY, Cheung SW, Cho P. Clinical performance of an orthokeratology lens fitted with the aid of a computer software in Chinese children. Contact Lens Anterior Eye. 2012;35:180-184.

50. Lum E, Golebiowski B, Swarbrick HA. Mapping the corneal sub-basal nerve plexus in orthokeratology lens wear using in vivo laser scanning confocal microscopy. Invest Ophthalmol Vis Sci. 2012;53;1803-1809. 